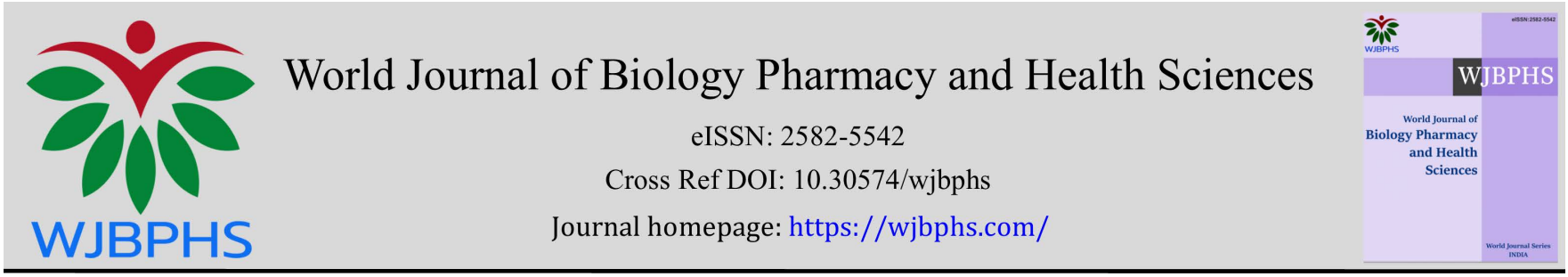

(RESEARCH ARTiCLE)

\title{
Design of primers in the molecular detection of Feline Panleukopenia Virus
}

\author{
Cristóbal Heraldo Carreño, Carlos Osvaldo Navarro * and María Antonieta Jara \\ Department of Preventive Animal Medicine, Faculty of Veterinary and Livestock Sciences, FAVET, Universidad de Chile.
}

World Journal of Biology Pharmacy and Health Sciences, 2021, 08(03), 019-029

Publication history: Received on 25 November 2021; revised on 28 December 2021; accepted on 30 December 2021

Article DOI: https://doi.org/10.30574/wjbphs.2021.8.3.0129

\begin{abstract}
Feline Panleukopenia is a disease characterized by a reduction in the number of circulating leukocytes and enteritis with degeneration of the intestinal villi. The etiologic agent, called Feline Panleukopenia virus (FPV), belongs to the Parvoviridae family, is highly contagious and has high mortality and morbidity.
\end{abstract}

Although vaccination of healthy cats is the most effective way to prevent the disease, once the symptoms appear, the treatment is supportive, presenting high mortality in the first days of the disease.

FPV positive cats should be hospitalized and isolated for at least 2 weeks to avoid viral transmission. Early detection is usually done with the enzyme-linked immunoadsorption assay (ELISA) test that detects viral antigens in stool samples.

As a complementary diagnostic method, in this work it was proposed to implement the Polymerase Chain Reaction (PCR) aimed at the diagnosis of FPV initially in positive samples from two feline vaccines and one canine vaccine. As an approximation to real samples, the commercial vaccines were mixed with feces and blood from a healthy cat.

The results showed that the In Silico design was successful strategy based on the VP2 gene sequences of FPV available in GenBank® in conjunction with the sharp visualization of positive controls of the expected size and without amplification. nonspecific or negative controls. The fragments obtained has a nucleotide identity percentage greater than $97 \%$ with respect to the information available in Genbank ${ }^{\circledR}$ and corroborates the detection of a VP2 fragment.

Thus, the future option of applying the same protocol in a diagnostic way is opened, using samples obtained from suspected patients who are infected with FPV.

Keywords: In silico primers design; Diagnosis; VPF; Implementation

\section{Introduction}

Feline Panleukopenia Virus (FPV) is a parvovirus responsible for Feline Panleukopenia, a disease characterized by a reduction in the number of circulating leukocytes and enteritis with degeneration of intestinal villi. The virus has a worldwide distribution, is resistant to disinfection and can survive for several months in the environment. In addition, it is highly contagious and presents a high mortality and morbidity, being able to infect the domestic cat, among other species.

\footnotetext{
*Corresponding author: Carlos Navarro; E-mail: canavarr@uchile.cl

Department of Preventive Animal Medicine, Faculty of Veterinary and Livestock Sciences, FAVET, Universidad de Chile.. 
The most effective way to prevent disease is by vaccinating healthy cats. There are attenuated virus and live modified virus vaccines, generating protection from 3 years to life. However, once the symptoms appear, the treatment is supportive, presenting high mortality in the first days of the disease.

Cats that are diagnosed with FPV should be hospitalized and isolated for at least 2 weeks to avoid transmission of the virus. Early detection of FPV is usually done with tests based on the enzyme-linked immunosorbent assay (ELISA), which detects FPV antigens in stool samples.

These tests can be confirmed, if the test is negative and the cat still shows sign of panleukopenia, by Polymerase Chain Reaction (PCR) of a stool or blood sample. It is also useful to perform blood PCR when there is no diarrhea and therefore ELISA tests cannot be used. In summary, PCR is presented as a useful tool for the diagnosis of the disease, complementing those currently used in small animal clinics.

With this background, the implementation of a molecular diagnostic protocol that uses conventional PCR as a method to detect FPV present in both commercial vaccines and in the mixture with feces and blood from healthy cats was proposed in this. The success obtained suggests its prompt application in a diagnostic manner, using samples obtained from patients suspected of being infected with FPV.

\section{Background}

\subsection{Feline Panleukopenia virus}

The Parvoviridae family comprises the subfamilies: Parvovirinae, which infects vertebrates, and Densovirinae, which infects invertebrates. The Parvovirinae subfamily includes eight genera, where the genus Protoparvovirus is found, which includes the Feline Panleukopenia Virus (FPV) [1,2,3]

FPV is the parvovirus responsible for Feline Panleukopenia, a disease characterized by a severe reduction in the white blood cell count and enteritis with degeneration of the intestinal villi [4,5]. Research on viral agents associated with feline reproductive problems reveals a high association with feline panleukopenia virus. It corresponds to a small (20 $\mathrm{nm}$ ), non-enveloped, single-stranded DNA genome virus of around 5000 bases, which encodes for two capsid proteins (VP1 and VP2) and two non-structural proteins (NS1 and NS2). The virus replicates using host cell polymerase, where the NS1 protein has a role in regulating replication. The VP1 and VP2 proteins assemble to produce the capsid, which corresponds $90 \%$ to VP2 and 10\% to VP1, where the unique region of VP1 contains functions for cellular infection, including phospholipase A2 activity. The capsid controls receptor binding and the cellular infection process and is also the primary target of host antibodies. The NS2 protein participates in virus assembly and nuclear transport, although its role is not yet clear [1,6]. Historically, there have been minimal changes in the FPV genome, even a study indicates that there have been no changes in the amino acid sequence of VP2, indicating a lack of appearance of new variants $[7,8,9]$. The virus has a worldwide distribution, is resistant to disinfection and can survive for several months in the environment. Furthermore, it is highly contagious and presents a high mortality and morbidity, being able to infect the domestic cat and other species of the Felidae, Mustelidae, Procyonidae and Viverridae family $[4,10,11,12]$. There is a close relationship between FPV and Canine Parvovirus type 2 (CPV-2), due to the emergence of the latter from evolution from FPV, through the acquisition of five or six amino acid changes in the protein gene of the capsid. This leads to both viruses having similar genetic and structural characteristics $[13,14,15,16]$.

\subsection{FPV pathogenesis}

FPV causes a systemic infection, mainly in unvaccinated or poorly vaccinated cats younger than 1 year. Transmission occurs via the fecal-oral route, and indirectly through fomites, which represent the main route of transmission. FPV enters the cell using transferrin receptors and replicates in cells that are in the S phase of the mitotic cycle. Initially, 1824 hours after infection, the virus replicates in lymphoid tissue of the oropharynx and after 2-7 days it spreads via the blood to all tissues $[4,11,14]$.

Infection of the lymphoid tissue leads to the necrosis of this tissue, causing immunosuppression. Lymphopenia can occur directly through lymphocytolysis, but also indirectly, as a result of the migration of lymphocytes to the tissues. In the bone marrow, viral replication occurs in early progenitor cells, explaining the dramatic effect on myeloid cell populations, which is reflected in panleukopenia. The replication of the virus in the crypts of intestinal epithelial cells causes shortening of the villi, increased intestinal permeability, and malabsorption $[4,11,14]$. 
In utero infection in early pregnancy can result in stillbirth, reabsorption, abortion, and mummification. In late pregnancy, FPV can cause damage to neuronal tissue, interfering with the cortical development of the cerebellum and causing abnormalities in the optic nerve and retina. Within the affected litter, some pups may be clinically healthy, probably due to innate resistance or acquisition of maternal antibodies. Still, these pups can carry the virus for up to 2 months after birth $[4,11]$.

Fecal elimination of the virus usually lasts for several days, and in some cats it can persist for up to 6 weeks [11,17].

Clinical signs of the FPV. Not all FPV-infected cats develop clinical signs, and the severity of these depends on age, immune status, and concomitant infections. The most common clinical signs are nonspecific, such as fever, depression, and anorexia. Sometimes vomiting unrelated to food occurs, and less commonly, diarrhea develops, which changes from watery to hemorrhagic during the disease. Cats generally die of complications associated with sepsis, dehydration, and disseminated intravascular coagulation (DIC) $[4,14,18]$.

In newborns, the main signs of FPV infection are neurological, with ataxia, hypermetry, and blindness. There may also be signs of cerebellar dysfunction, such as incoordination and tremor $[4,11,19]$.

\subsection{FPV treatment}

Cats diagnosed with FPV should be hospitalized and isolated for at least 2 weeks to avoid transmission of the virus. The treatment is supportive, applying fluid therapy to maintain hydration, and antibiotic therapy to prevent sepsis caused by the entry of bacteria by a destroyed gastrointestinal barrier. Antiemetics can also be administered if vomiting occurs, and it is important to restart oral and food intake as soon as possible $[11,14,20,21]$.

\subsection{Immunization}

Colostrum antibodies to feline panleukopenia have a half-life of 9.5 days. Cat pups can be passively immunized by injecting anti-FPV serum in case of outbreaks or places where infection is present. This immunization is quick and lasts 2-4 weeks, however, its prophylactic effectiveness has only been confirmed in dogs $[4,14,18]$.

The most effective way to prevent disease is by vaccinating healthy cats. There are attenuated virus and live modified virus vaccines, generating protection from 3 years to life. Caution should be exercised with immunosuppressed animals, as there have been cases in which immunization with feline live virus vaccine manifests the picture of feline panleukopenia $[4,11,22]$.

In Chile, there are two vaccines authorized by the Agricultural and Livestock Service (SAG) for marketing: Feligen CRP and Felocell 3. Both contain strains of Herpesvirus, Calicivirus and FPV viruses. The Feligen CRP vaccine contains attenuated virus strains, while the Felocell 3 vaccine contains live modified viruses [23].

\subsection{Diagnosis}

In general, the clinical signs, hematological changes, and postmortem findings are characteristic and enough for a presumptive diagnosis of feline panleukopenia [21,24].

To confirm this diagnosis, FPV can be diagnosed by viral isolation by cell culture, however this is not a routine procedure. Early detection of FPV is usually done with tests based on the enzyme-linked immunosorbent assay (ELISA), which detects FPV antigens in stool samples. It has also been observed that the CPV tests have an acceptable sensitivity and specificity to detect FPV $[11,14,25,26]$.

These tests can be confirmed, in case the test is negative, and the cat still shows signs of panleukopenia, by PCR of a stool or blood sample. It is also useful to perform blood PCR when there is no diarrhea, and therefore, ELISA tests cannot be used due to the absence of samples [4,14,27].

\section{Material and methods}

This work was carried out in the Animal Virology and Microbiology laboratories of the Department of Preventive Animal Medicine, belonging to the Faculty of Veterinary and Livestock Sciences of the University of Chile. 


\subsection{Samples}

For the PCR reaction, two stool and blood samples obtained from a healthy cat were used, to which the Feline Panleukopenia Virus obtained from the commercial vaccines Feligen CRP (V1) and Felocell 3 (V2) will be added. The two vaccines mentioned will be used as a positive control, and DNA from viruses and bacteria available in the laboratory will be used as a negative control, including Canine herpes virus, Brucella canis and Pseudomonas aeruginosa. Nucleasefree water will be used to control reagents. Additionally, the PCR reaction will be carried out on a Canine Parvovirus sample, obtained from the Nobivac® DHPPI vaccine (V3).

\subsection{Extraction of DNA}

The extraction of the viral DNA was carried out through the protocol used [23]. This protocol involves boiling the virus sample for 10 minutes, and then chilling on ice. The result of this process was diluted in distilled water at a ratio of $1: 10$ to avoid the action of PCR inhibitors.

\subsection{Primers}

The Genbank $®$ database was used to obtain 56 official sequences of the FPV VP2 gene. Subsequently, using the free access software Clustal $\Omega$, the sequences were aligned, in order to determine candidate nucleotide identity zones to be used for the design of the primers. This design contemplated the use of free access software OligoPerfect Design from Thermofisher Scientific ${ }^{\circledR}$

These primers were subjected to the BLAST program, in order to generate greater specificity against the target area of the sequence. Other parameters were also considered, choosing those with a GC percentage close to $50 \%$ and a Tm difference of no more than $3{ }^{\circ} \mathrm{C}$. Once the sequence of the primers was obtained, their synthesis was commissioned from IDT® via Fermelo, Chile.

\subsection{PCR reaction mix}

To carry out the PCR reaction, $15 \mu \mathrm{L}$ of the commercial 2X PCR Master Mix kit (Taq DNA polymerase, MgCl2 and deoxyribonucleotide triphosphates) were used. $5 \mu \mathrm{L}$ of the template DNA and $5 \mu \mathrm{L}$ of each specific primer $(0.1 \mathrm{uM})$ were used, thus completing a total volume of $30 \mu \mathrm{l}$.

\subsection{PCR reaction}

The PCR reaction was carried out in a $0.2 \mathrm{~mL} 92$-well Apollo thermocycler, and the following protocol was used: activation of Taq polymerase in one step at $94^{\circ} \mathrm{C}$ for 2 minutes. Then, 40 denaturation cycles were performed at $94^{\circ} \mathrm{C}$ for 30 seconds; Alignment of primers at $55^{\circ} \mathrm{C}$ for 1 minute and polymerization at $72^{\circ} \mathrm{C}$ for 1 minute. Finally, the extension stage was carried out at $72^{\circ} \mathrm{C}$ for 10 minutes. The alignment temperature was previously determined by means of a temperature gradient thermocycler.

\subsection{Visualization of the amplified product}

To visualize the product of the PCR reaction, a $2 \%$ agarose gel electrophoresis was performed in Tris-borate buffer (90 Mm Tris-borate, $10 \mathrm{mM}$ EDTA). The PCR reaction product was mixed in a 6: 1 ratio with a commercial filler (6X Mass Ruler Loading Dye Solution (Fermentas®).

Electrophoresis was performed at $90 \mathrm{~V}$ for 40 minutes and a standard with fragments between 100 and $3000 \mathrm{bp}$ (Fermentas $($ ) was used as a molecular weight marker. After the 40 minutes had elapsed, the agarose gel was incubated in ethidium bromide $(0.5 \mu \mathrm{g} / \mathrm{ml}$ ) (Fermelo®) for 30 minutes at room temperature. After incubation, the gel was placed in an ultraviolet light transilluminator (UVP® Transilluminator), where it was photographed to record the results.

\subsection{Determination of the identity of the amplified product}

Sample 8, one of the positive results (in triplicate) was sent without purification to the company Genytec Ltda, to determine the nucleotide sequence. After this, the three nucleotide sequences obtained were aligned using the Clustal $\Omega$ software, to obtain a consensus sequence.

The consensus sequence was entered into the BLAST program, to determine within the database, with which sequences the greatest nucleotide identity is presented. 
Finally, the sample that, in the PCR, originates a DNA fragment of size greater than 600 bp and whose nucleotide sequence entered the BLAST program, obtained values over $90 \%$ nucleotide identity with respect to the available FPV VP2 gene sequences, was considered positive.

\subsection{Biosafety}

In the laboratory, the following were considered: limited access to the facilities, use of a clean and closed apron, use of clean material, and adequate waste disposal. Latex gloves were used for the subsequent PCR and electrophoresis procedure, both to avoid contamination of the sample and to manipulate substances such as ethidium bromide, which has mutagenic properties. When using the transilluminator, UV filter glasses and an acrylic plate located between the equipment and the person viewing the gel were used. Finally, the gel and gloves used were incinerated.

\section{Results}

\subsection{Implement a conventional PCR assay for the molecular diagnosis of FPV}

\subsubsection{Sequences used}

A total of 56 official FPV VP2 gene sequences were obtained, which were aligned with the Clustal $\Omega$ software, determining nucleotide identity zones (Annex 1).

\subsubsection{Primers}

The primers were chosen and synthesized: F1: TGGTTGATGCAAATGCTTGGG and F2: AACCAACCTCAGCTGGTCTC, which generate an amplicon of $681 \mathrm{bp}$ (Annex 2).

\subsubsection{PCR reaction}

In Figure 1 it is possible to observe the bands originated in the PCR reaction of the controls, where clear bands were observed both in the cases of positive controls and for each one of the respective stool (feces) and blood samples mixed with the vaccine and in the V3 vaccine. No bands were observed in the negative controls or in the reagent control. As a reference, a marker of molecular size between 100 and 3000 bp was used.

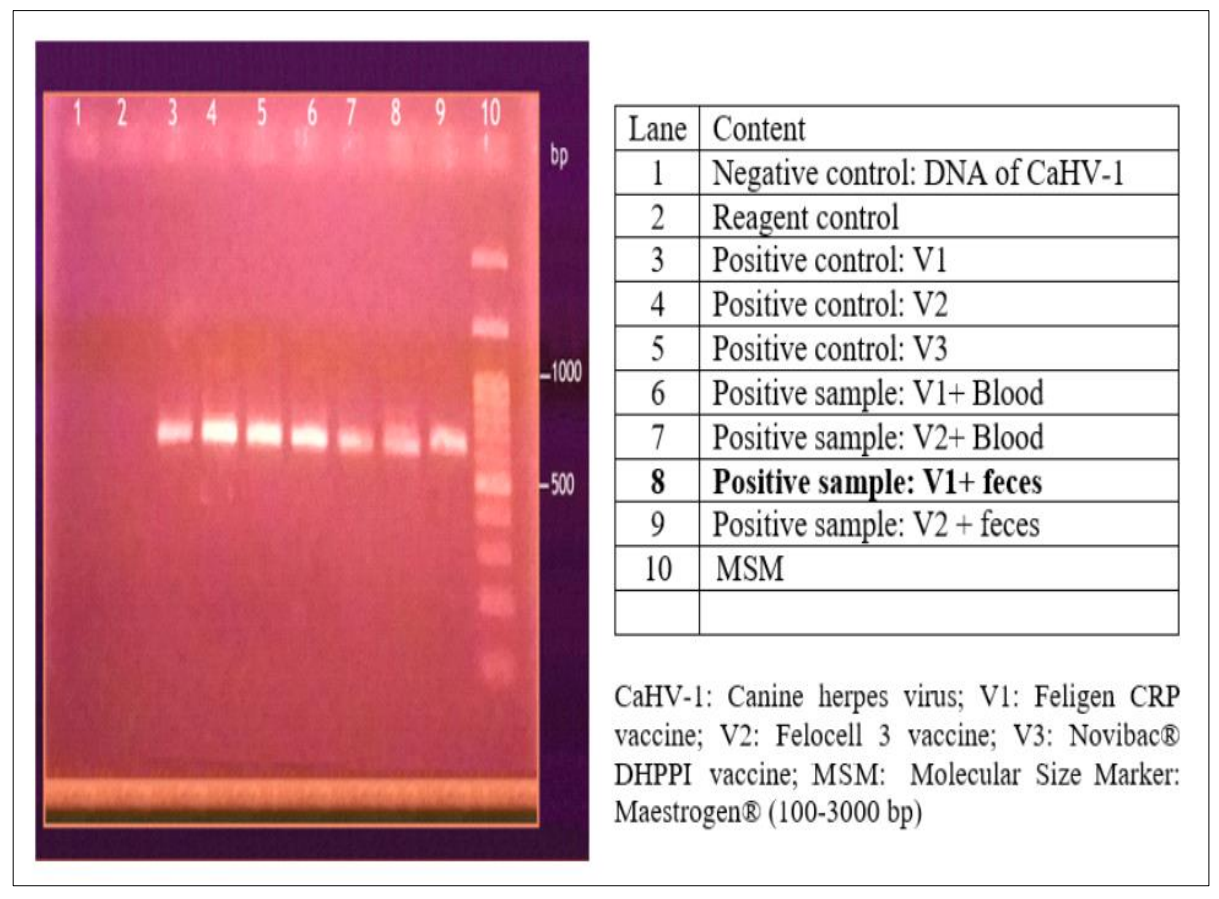

Figure 1 Electrophoresis (Agarose 2\%) 


\subsection{Identify the PCR product by percentage of nucleotide identity (PNI) with respect to official Genbank® data}

To determine the sequence of the amplified fragment, the PCR product from sample 8 was sent to the company Genytec Ltda and the alignment of the sequences obtained allowed defining a consensus sequence: CHCJ (annex 3) and later its percentage of nucleotide identity with respect to sequences stored in the Genbank® (Figure 2).

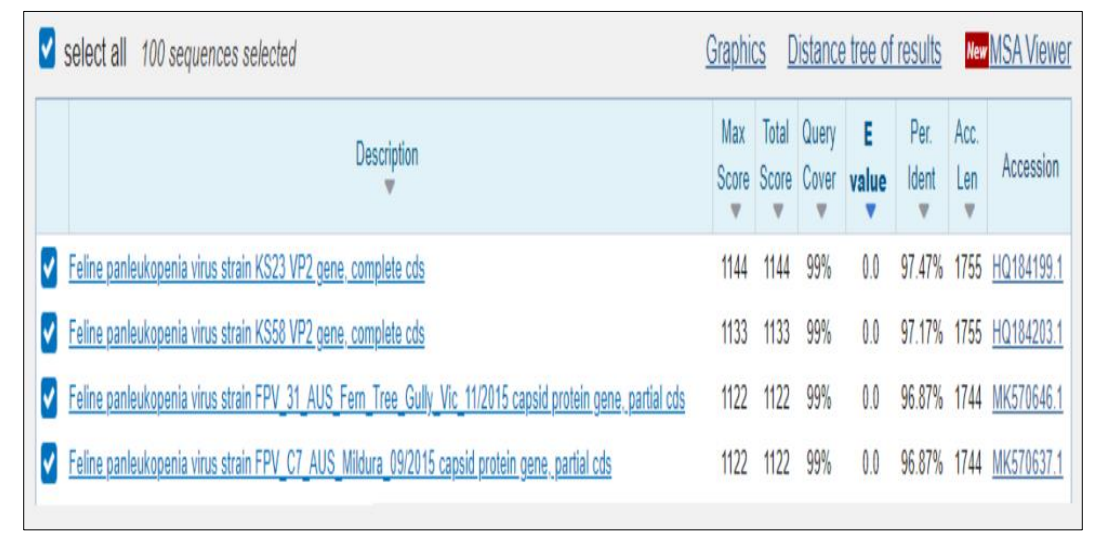

Figure 2 Partial visualization of the percentage of nucleotide identity of the CHCJ sequence respect to the first 100 similar sequences according to BLAST. PNI>96\% (The extended result in relation to the PIN obtained is presented in Annex 6.)

\section{Discussion}

Although clinical signs, hematological changes, and postmortem findings are characteristic and enough for a presumptive diagnosis of feline panleukopenia [2], to confirm this diagnosis, FPV can be diagnosed by viral isolation through cell culture. However, this is not a routine procedure, primarily due to the length of the procedure. Early detection of FPV uses some tests based on enzyme-linked immunoadsorption (ELISA) that detects FPV antigens in feces. Furthermore, these tests have acceptable sensitivity and specificity values to detect FPV [4,11,25]. However, for some years it has been recommended to have a complementary diagnostic method with sensitivity and specificity values such as those attributable to the Polymerase Chain Reaction $[28,29,30]$.

In this context, the molecular diagnosis of FPV based on a conventional PCR protocol with the use of the In silico design of primers or primers thanks to free access software and validate the identity of the DNA fragment has been proposed in this work.

Thus, the results obtained suggest that the use of at least 56 official FPV VP2 nucleotide sequences stored in the Genbank $®$ was a successful initial strategy, since the consensus sequence obtained - around 1800 nucleotides - turned out to be a good candidate as a template for the design of the partitions, in which those areas that did not present common spaces or that did not allow finding 20 nucleotide primers or primers were excluded.

Subsequently, the choice of primers according to the Oligoperfect Design from Thermofisher Scientific $®$ software was successful, since DNA fragments greater than $600 \mathrm{bp}$ were obtained, which is consistent with that indicated by the chosen software $(\approx 680 \mathrm{bp})$.

The PCR technique implemented does not generate nonspecific bands in the positive controls in the $2 \%$ agarose gel, which corroborates an effective choice of the primers or primers, validated by the null presence of DNA fragments or amplicons when using negative controls or nuclease free water.

Consequently, the sample sent to be sequenced in triplicate indicated a high percentage of nucleotide identity (PIN $>97$ ) with respect to the official sequences of the Genbank $₫$ and allows us to suggest that the DNA fragment obtained corresponds to a segment of the VP2 gene of FPV (annex 4). 
Finally, the results obtained would suggest that the strategy of choosing a common area - present in 56 nucleotide sequences of the VP2 gene of FPV as a possible candidate for the In Silico primers design was successful, corroborated both by a clear and specific detection and by sequencing of the expected DNA fragment.

\section{ANNEX 1}

Consensus sequence from 56 FPV sequences (in yellow) according to Genbank®. The common areas, candidates for the design of primers are indicated in green.

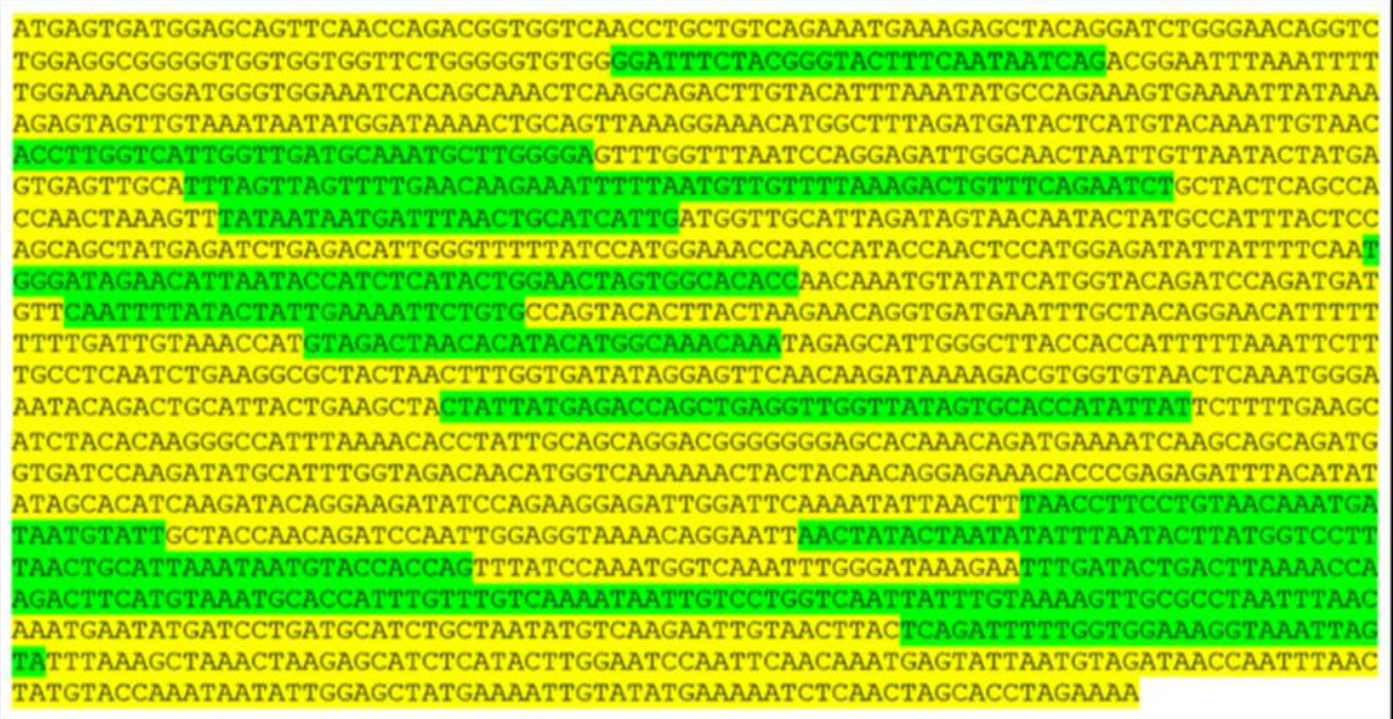

\subsection{ANNEX 2}

Primers design, according to Oligoperfect Design from Thermofisher Scientific ${ }^{\circledR}$

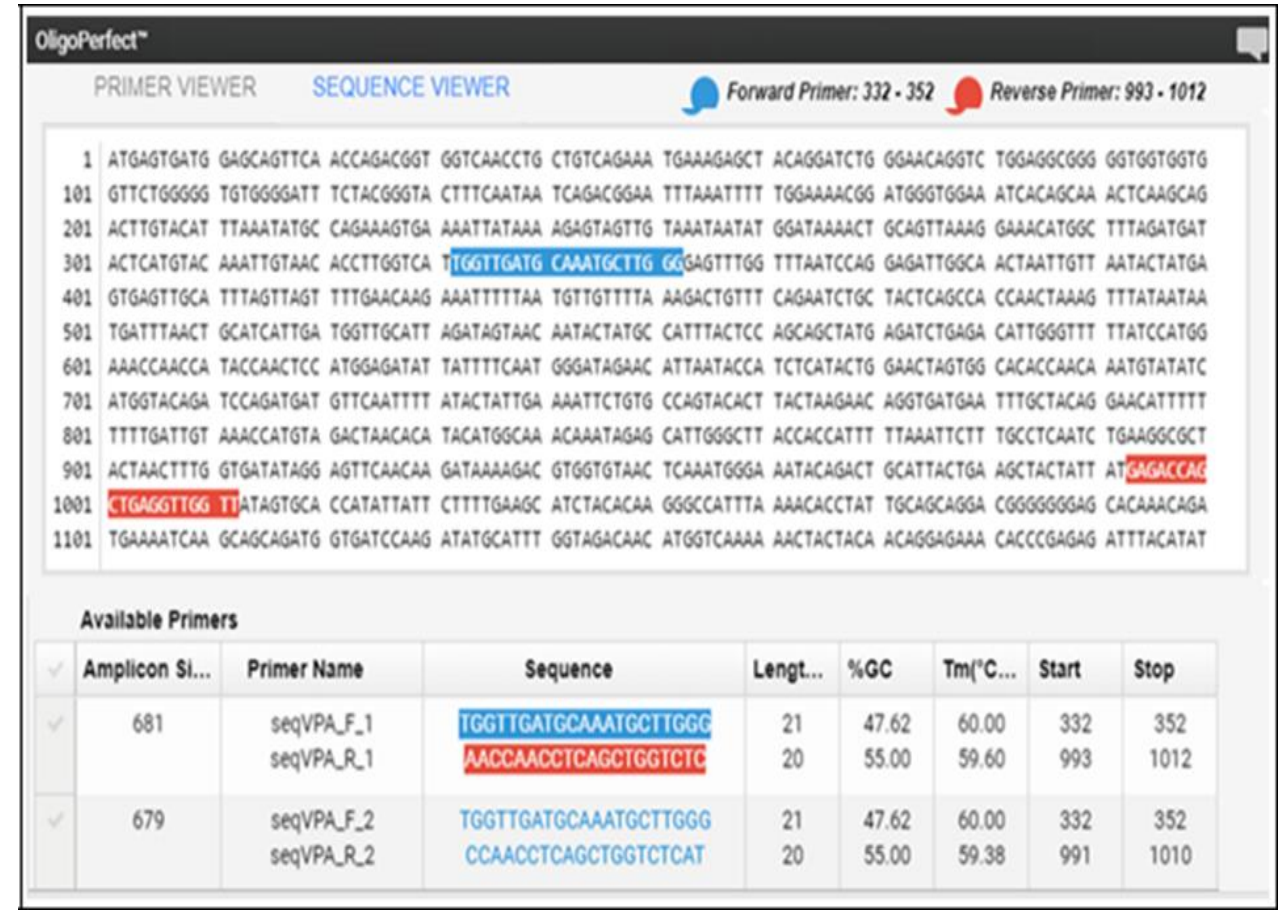

\subsection{ANNEX 3}

Alignment of sequences from Genytec Ltda. (Sample 8) and CHCJ consensus sequence. 


\begin{tabular}{|c|c|c|}
\hline Seq3 & GTTGATGCAAATGCTTGGGGAGTTTGGTTTAATCCAGGAGATTGGCAACTAATTGTTAAT & 60 \\
\hline Seq1 & GTTGATGCAAATGCTTGGGGAGTTTGGTTTAATCTAGGAGATTGGCAACTAATTGTTAAT & 60 \\
\hline seq2 & -TTGATGCAAATGCTTGGGGAGTTTGGTTTAACCCAGGAGATTGGCAACTAATTATTAAT & 59 \\
\hline Seq3 & ACTATGAGTGAGTTGCATTAAGTTAGTTTTGAACAAGAAATTTTTAATGTTGTTTTAAAG & 120 \\
\hline Seq1 & ACTATGAGTGAGTTGCATTAAGTTAGTTTTGAACAAGAAATTTTTAATGTTGTTTTAAAG & 120 \\
\hline Seq2 & 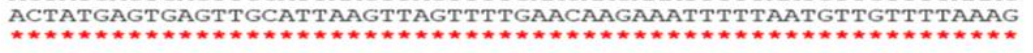 & 119 \\
\hline Seq3 & АСTGTTTCAGAATCTGCTACTCAGCCACCAACTAAAGTTTATAATAATGATTTAACTGCA & 180 \\
\hline Seq1 & ACTGTTTCAGAATCTGCTACTCAGCCACCAACTAAAGTTTATAATAATGATTTAACTGCA & 180 \\
\hline Seq2 & 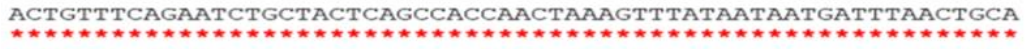 & 179 \\
\hline seg 3 & TCATTGATGGTTGCATTAGACAGTAACAATACTATGCCATTTACTCCAGCAGCTATGAGA & 240 \\
\hline Seq1 1 & TCATTGACACATGCATTAGATAGTAACAATACTATGCCATTTACTCTAGCAGCTATGAGA & 240 \\
\hline Seq2 2 & 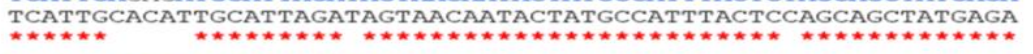 & 239 \\
\hline $\mathrm{seq} 3$ & TATGAGACATTGGGTTTTTATCCATGCACACCAACCATACCAACTCCATGGAGATATTAT & 300 \\
\hline Seq1 & TATGAGACATTGGGTTTTTATCCATGGAAACCAACCATACCAACTCCATGGAGATATTAT & 300 \\
\hline seq2 & 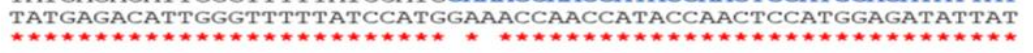 & 299 \\
\hline seq3 & TTTCAATGGGATAGAACATTAACACCATCTCATACTGGAACTAGTGGCACACCAACAAAT & 360 \\
\hline Seq1 & TTTCAATGGGATAGAACATTAACACCATCTCATACTGGAACTAGTGGCACACCAACAAAT & 360 \\
\hline Seq2 & 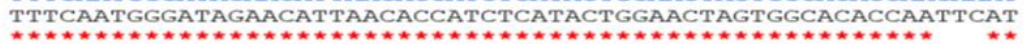 & 359 \\
\hline seg3 3 & 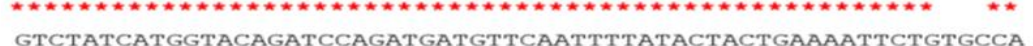 & 420 \\
\hline Seg1 1 & 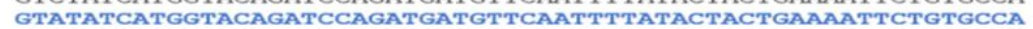 & 420 \\
\hline seq2 & $\begin{array}{l}\text { GTATATCATGGTACAGATCCAGATGATGTTCAATTTTATACTACTGAAAATTCTGTGCCA } \\
\star \star \star \\
\text { M }\end{array}$ & 419 \\
\hline seg 3 & GTACACTTACTAAGAACAGGTGATGAATTTGCTACAGGAACATTTTTTTTTGATTGTAAA & 480 \\
\hline seq1 & GTACACTTACTAAGAACAGGTGATGAATTTCCTACAGGAACATTTTTTTTTGATTGTAAA & 480 \\
\hline seq2 & 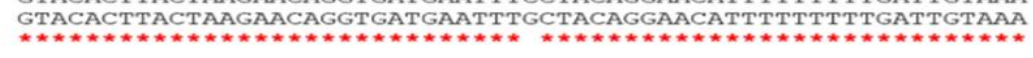 & 479 \\
\hline seg 3 & CСATCTAGACTAACACATATTCGGCAAACAAATAGAGCATTGTGCTTACCACCATTTTTA & 540 \\
\hline & CCATCTAGACTAACACATACATGGCAAACAAATAGAGCATTGGGCTTACCACCATTTTTA & 540 \\
\hline \multirow[t]{2}{*}{ Seq2 } & CCATCTAGACTAACACATACATGGCAAACAAATAGAGCATTGGGCTTACCACCATTTTTA & 539 \\
\hline & 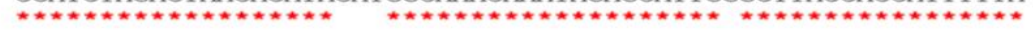 & \\
\hline Seg 3 & АATTCTTTGCСTCAATCTGAAGGCGCTACTAACTTTGGTGATATAGGAGTTCAACAAGAT & 600 \\
\hline Seq1 1 & AATTCTTTGCCTCAATCTGAAGGCGCCСTTAACTTTGGTGATATAGGAGTTCAACAAGAT & 600 \\
\hline \multirow[t]{2}{*}{ seq2 } & AATTCTTTGCCTCAATCTGAAGGCGCCCTTAACTTTGGTGATATAGGAGTTCAACAAGAT & 599 \\
\hline & 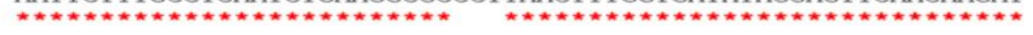 & \\
\hline seg 3 & AAAAGACGTGGTGTAACTCAAATGGGAAATACAGACTGCATTACTGCCGCGACTATTATG & 660 \\
\hline Seq1 & AAAAGACGTGGTGTAACTCAAATGGGAAATACAGACTGCATTACTGCCGCGACTATTATG & 660 \\
\hline \multirow[t]{2}{*}{ Seq2 } & AAAAGACGTGGTGTAACTCAAATGGGAAATACAGACTGCATTACTGCCGCGACTATTATG & 659 \\
\hline & & \\
\hline Seq3 3 & AGACCAGCTGAGG-- 673 & \\
\hline Seq1 & AGACCAGCTGAAAT- & \\
\hline Seq2 2 & AGACCAGCTGAAATT 674 & \\
\hline
\end{tabular}

\section{Consensus sequence (CHCJ)}

$>\mathrm{CHCJ}$

GTTGATGCAAATGCTTGGGGAGTTTGGTTTAATCCAGGAGATTGGCAACTAATTGTTAATACTATGAGTGAGTTGCAT TAGTTAGTTTTGAACAAGAAATTTTTAATGTTGTTTTAAAGACTGTTTCAGAATCTGCTACTCAGCCACCAACTAAAG TTTATAATAATGATTTAACTGCATCATTGACACTTGCATTAGATAGTAACAATACTATGCCATTTACTCCAGCAGCTA TGAGATATGAGACATTGGGTTTTTATCCATGGAAACCAACCATACCAACTCCATGGAGATATTATTTTCAATGGGATA GAACATTAACACCATCTCATACTGGAACTAGTGGCACACCAATTCATGTATATCATGGTACAGATCCAGATGATGTTC AATTTTATACTACTGAAAATTCTGTGCCAGTACACTTACTAAGAACAGGTGATGAATTTGCTACAGGAACATTTTTTTT TTGATTGTAAACCATCTAGACTAACACATACATGGCAAACAAATAGAGCATTGGGCTTACCACCATTTTTAAATTCTT TGCCTCAATCTGAAGGCGCCCTTAACTTTGGTGATATAGGAGTTCAACAAGATAAAAGACGTGGTGTAACTCAAATGG GAAATACAGACTGCATTACTGCCGCGACTATTATGAGACCAGCTGAAAT

\subsection{ANNEX 4}

Comparison between the first and last sequences from BLAST program with respect to HCCJ sequence. 
World Journal of Biology Pharmacy and Health Sciences, 2021, 08(03), 019-029

\begin{tabular}{|c|c|c|c|c|c|c|c|c|}
\hline$\nabla$ select all 100 sequences selected & \multicolumn{2}{|c|}{ GenBank Graphics } & \multicolumn{4}{|c|}{ Distance tree of results } & \multicolumn{2}{|c|}{ Nen MSAViewer } \\
\hline Descipition & Scientific Name & $\begin{array}{l}\text { Max } \\
\text { Score S }\end{array}$ & & $\begin{array}{l}\text { Query } \\
\text { Cover }\end{array}$ & $\begin{array}{c}\text { E } \\
\text { value } \\
\nabla\end{array}$ & $\begin{array}{l}\text { Per. } \\
\text { Ident } \\
\nabla\end{array}$ & $\begin{array}{l}\text { Acc. } \\
\text { Len } \\
\nabla\end{array}$ & Accession \\
\hline V Feline parleukcopenia virus strain KS23 VP2 gene, complete cds & Feline parleukop. & 1144 & 1144 & $99 \%$ & 0.0 & $97.47 \%$ & 1755 & H0184199.1 \\
\hline 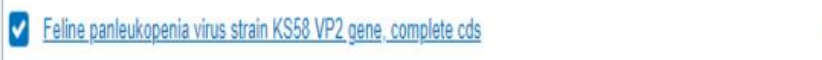 & Feline parleukoo. &.. .1133 & 1133 & $99 \%$ & 0.0 & $97.17 \%$ & 1755 & H018420203.1. \\
\hline 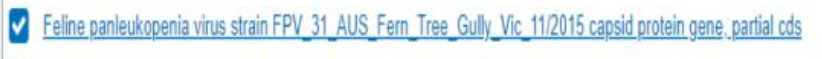 & Feline parleukop. & 1122 & 1122 & $99 \%$ & 0.0 & $96.87 \%$ & 1744 & MK570646.1 \\
\hline 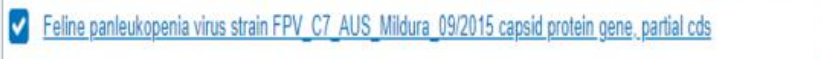 & Feline parleukop. & 1122 & 1122 & $99 \%$ & 0.0 & $96.87 \%$ & 1744 & MK570637.1 \\
\hline 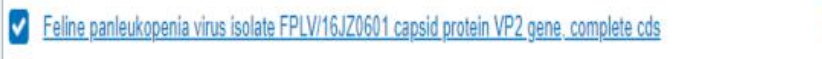 & Feline parleukop. & 1122 & 1122 & $99 \%$ & 0.0 & $96.87 \%$ & 1755 & MK671154.1 \\
\hline V Feline panleukopenia virus isolate UL-0416 capsid protein VP2 gene, complete cds & Feline parleukop. & 1122 & 1122 & $99 \%$ & 0.0 & $96.87 \%$ & 1755 & MF541125.1 \\
\hline 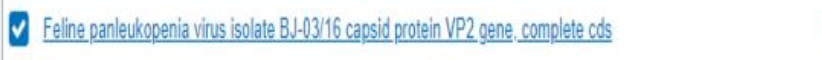 & Feline parleukop. & 1122 & 1122 & $99 \%$ & 0.0 & $96.87 \%$ & 1755 & MF541120.1 \\
\hline 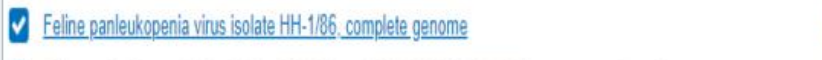 & Feline panleukop. & 1122 & 1122 & $99 \%$ & 0.0 & $96.87 \%$ & 5123 & KX900570.1 \\
\hline 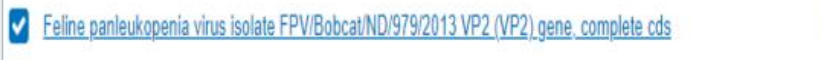 & Feline parleukop. & 1122 & 1122 & $99 \%$ & 0.0 & $96.87 \%$ & 1755 & k.813993.1 \\
\hline Veline paneleukopenia virus strain K23 VP2 gene.complete cds & Feline parleukop. & 1122 & 1122 & $99 \%$ & 0.0 & $96.87 \%$ & 1755 & Ho1841941. \\
\hline 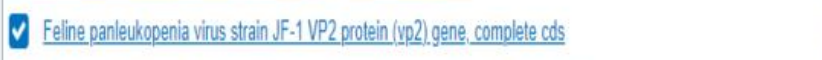 & Feline parleukop. & 1122 & 1122 & $99 \%$ & 0.0 & $96.87 \%$ & 1755 & $\underline{004742361}$ \\
\hline 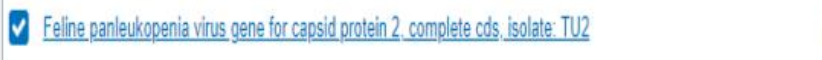 & Feline parleukop. & 1122 & 1122 & $99 \%$ & 0.0 & $96.87 \%$ & 1755 & ABD00066.1 \\
\hline 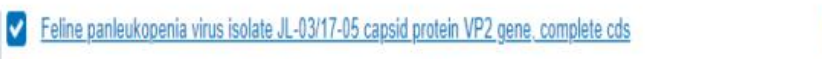 & Feline panleukoo. &.. .1110 & 1110 & $99 \%$ & 0.0 & $96.57 \%$ & 1755 & MF541124.1. \\
\hline 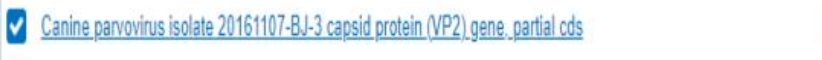 & Canine pavovivis & 81110 & 1110 & $99 \%$ & 0.0 & $96.57 \%$ & 1752 & MF547717.1 \\
\hline$\checkmark$ Canine parvovirus cansid protein (VP2).gene.partial cds & Canine parovivis & 11110 & 1110 & $99 \%$ & 0.0 & $96.57 \%$ & 1755 & MG462710.1 \\
\hline 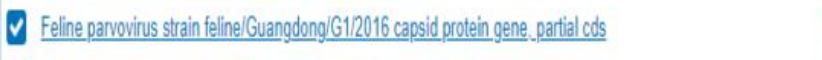 & Feline parvovirus & 1110 & 1110 & $99 \%$ & 0.0 & $96.57 \%$ & 1676 & kY4551727.1. \\
\hline 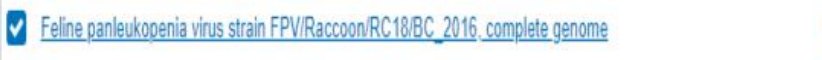 & Feline panleukoo. & 1110 & 11110 & $99 \%$ & 0.0 & $96.57 \%$ & 4528 & MF069447.1. \\
\hline 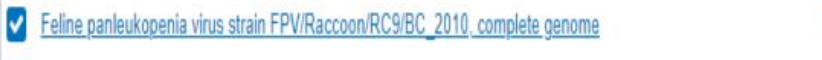 & Feline panleukop. & 1110 & 1110 & $99 \%$ & 0.0 & $96.57 \%$ & & MF069446.1 \\
\hline 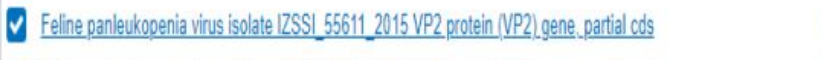 & Feline panleukop. & 1110 & 1110 & $99 \%$ & 0.0 & $96.57 \%$ & & $\underline{x \times 943318.1}$ \\
\hline 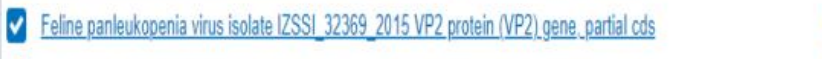 & Feline panleukoo. & 1110 & 1110 & $99 \%$ & 0.0 & $96.57 \%$ & & kx943315.1 \\
\hline 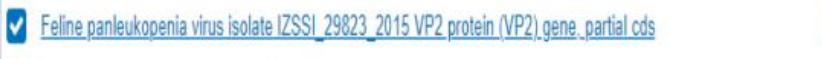 & Feline panleukop. & 1110 & 1110 & $99 \%$ & 0.0 & $96.57 \%$ & & $\underline{x \times 943314.1}$ \\
\hline V Feline panleukooenia virus strain HN-Z21. complete genome & Feline panlevkop. & 1110 & 1110 & $99 \%$ & 0.0 & $96.57 \%$ & 4688 & KX685354.1 \\
\hline$\checkmark$ Feline panleukoopenia virus strain $245-1478$ FPLV BADGER 2019 ITALYY capsid protein VP2 gene, complete cds & Feline panleukoo. & 1110 & 1110 & $99 \%$ & 0.0 & $96.57 \%$ & & $\underline{\text { MT274378.1 }}$ \\
\hline V Feline parvovirus isolate HF1. complete genome & Feline parvovins & 1110 & 1110 & $99 \%$ & 0.0 & $96.57 \% 5$ & & MT643656.1 \\
\hline
\end{tabular}

\section{Conclusion}

The developed PCR protocol can be suggested as a basis for the molecular diagnosis of FPV.

\section{Compliance with ethical standards}

\section{Acknowledgments}

We thank to Dr. Aron Mosnaim from the Wolf Foundation and Chicago Medical School, USA.

\section{Disclosure of conflict of interest}

The authors declare no conflict of interest 


\section{References}

[1] Hoelzer K, Parrish C. Evolution and Variation of the Parvoviruses. In: Domingo, E.; Parrishm C.; Holland, J. Origin and Evolution of Viruses. Second Edition. Academic Press. London, United Kingdom. 2008; 394-416.

[2] Maclachlan J, Dubovi E, Barthold S, Swayne D, Winton J. Parvoviridae. In: Fenner's Veterinary Virology. Fifth Edition. Academic Press. London, United Kingdom. 2017; 245-257.

[3] ICTV. International Committee On Taxonomy Of Viruses. 2020. Virus Taxonomy: 2019.

[4] Stuetzer B, Hartmann K. Feline parvovirus infection and associated diseases. Vet J. 2014; 201(2): 150-155.

[5] Pristo de Medeiros V, Alves de Carvalho D, Pereira H, Ferreira G, Rocha C, Pérez C, Kurissio J, Araújo J, Azevedo de Paula J. Research on viral agents associated with feline reproductive problems reveals a high association with feline panleukopenia virus. Vet Animal Sci. 2018; 6: 75-80.

[6] Truyen U, Parrish C. Feline panleukopenia virus: its interesting evolution and current problems in immunoprophylaxis against a serious pathogen. Vet Microbiol. 2013; 165(1-2): 29-32.

[7] Decaro N, Desario C, Miccolupo A, Campolo M, Parisi A, Martella V, Amorisco F, Lucente M, Lavazza A, Buonavoglia C. Genetic analysis of feline panleukopenia viruses from cats with gastroenteritis. J Gen Virol. 2008; 89(9): 22902298.

[8] Lamm C, Rezabek G. Parvovirus infection in domestic companion animals. Vet Clin North Am Small Anim Pract. 2008; 38(4): 837-850.

[9] Akkutay-Yoldar Z, Taylan Koç B. Comparison of partial and full VP2 gene sequences of parvovirus from domestic cats in Turkey. Veterinaria México OA. 2019.

[10] Greene C. Panleucopenia felina. In: Enfermedades infecciosas en perros y gatos. 2th edition Mcgraw-Hill. New York, USA. 2000; 56-63.

[11] Sykes J. Feline Panleukopenia Virus Infection and Other Virals Enteritides. In: Canine and Feline Infectious Diseases. Saunders. Missouri, United States. 2014; 187-194.

[12] Inthong N, Sutacha K, Kaewmongkol S, Sinsiri R, Sribuarod K, Sirinarumitr K, Sirinarumitr T. Feline panleukopenia virus as the cause of diarrhea in a banded linsang (Prionodon linsang) in Thailan J Vet MedSci. 2019.

[13] Horiuchi M, Yamaguchi Y, Gojobori T, Mochizuki M, Nagasawa H, Toyoda Y, Ishiguro N, Shinagawa M. Differences in the evolutionary pattern of feline panleukopenia virus and canine parvovirus. Virology. 1998; 249(2): 440452 .

[14] Truyen U, Addie D, Belák S, Boucraut-Baralon C, Egberink H, Frymus T, Gruffyd-Jones T, Hartmann K, Hosie M, Lloret A, Lutz H, Marsilio F, Pennisi M, Radford A, Thiry E, Horzinek M. Feline panleukopenia. ABCD guidelines on prevention and management. J Feline Med Surg. 2009; 11(7): 538-546.

[15] Möstl K, Addie D, Boucraut-Baralon C, Egberink H, Frymus T, Gruffyd-Jones T, Hartmann K, Hosie M, Lloret A Lutz H, Marsilio F, Pennisi M, Radford A, Thiry E, Truyen U, Horzinek M. Something old, something new: Update of the 2009 and 2013 ABCD guidelines on prevention and management of feline infectious diseases. J Feline Med Surg. 2015; 17(7): 570-582.

[16] Ahmed N, Riaz A, Zubair Z, Saqib M, Ijaz S, Nawaz-Ul-Rehman MS, Al-Qahtani A, Mubin M. Molecular analysis of partial VP-2 gene amplified from rectal swab samples of diarrheic dogs in Pakistan confirms the circulation of canine parvovirus genetic variant CPV-2a and detects sequences of feline panleukopenia virus (FPV). Virol J. 2018.

[17] Fooshee S. Feline Parvovirus infection. In: The Feline Patient. Wiley Blackwell, NJ 07030 USA. 2018.

[18] Hartmann K. Feline panleukopenia - update on prevention and treatment. Thai J VetMed Suppl. 2017.

[19] Garigliany M, Gilliaux G, Jolly S, Casanova T, Bayrou C, Gommeren K, Fett T, Mauroy A, Lévy E, Cassart D, Peeters D, Poncelet L, Desmecht D. Felinepanleukopenia virus in cerebral neurons of young and adult cats. BMC Vet Res. 2016.

[20] Awad R, Khalil W, Attallah A. Feline panleukopenia viral infection in cats: Application of some molecular methods used for its diagnosis. J Genet Eng biotechnol. 2018. 
[21] Day M, Horzinek M, Schultz R, Squires R. Guidelines for the vaccination of dogs and cats. J Small Animal Practice. 2016.

[22] SAG. Servicio Agrí́cola Y Ganadero. Medicamentos de uso veterinario. 2018.

[23] Desario E, Decaro N, Campolo M, Cavalli A, Cirone F, Elia G, Martella V, Lorusso E, Camero M, Bunavoglia C. Canine parvovirus infection: which diagnostic test for virus? J Virol Methods. 2005; 126(1-2): 179-185.

[24] Neuerer F, Horlacher K, Truyen U, Hartmann K. Comparison of different in-house test systems to detect parvovirus in faeces of cats. J Feline Med Surg. 2008; 10(3): 247-251.

[25] Nandi S, Sharma G, Gupta V, Deol P, Chander V, De UK, Gupta VK. Global Scenario of Canine Parvovirus Mutants: Epidemiology, Diagnostics and Immunoprophylactic Agents. JSM Vet Med Res. 2019.

[26] Balboni A, Bassi F, De Arcangeli S, Zobba R, Debola C, Alberti A, Battilani M. Molecular analysis of carnivore Protoparvovirus detected in white blood cells of naturally infected cats. BMC Vet Res. 2018.

[27] Streck A, Rüster D, Truyen U, Homeier T. An updated TaqMan real-time PCR for canine and feline parvoviruses. J Virol Methods. 2013; 193(1): 6-8.

[28] Faz M, Martínez J, Quijano-Hernández I, Fajardo R. Reliability of clinical diagnosis and laboratory testing techniques currently used for identification of canine parvovirus enteritis in clinical settings. J Vet Med Sci. 2017.

[29] Erlich H. Recent advances in the Polymerase Chain Reaction Science. 2019; 252: 1643-1650.

[30] Harris S. Optimization of the Polymerase Chain Reaction. Br J Biomed Sci. 1997; 54(3): 166-17. 